\title{
Jillese
}

\section{Characterizing the turbulent drag properties of rough surfaces with a Taylor-Couette set-up}

\author{
Pieter Berghout ${ }^{1, \dagger}$, Pim A. Bullee ${ }^{1,2}$, Thomas Fuchs ${ }^{3}$, Sven Scharnowski ${ }^{3}$, \\ Christian J. Kähler ${ }^{3}$, Daniel Chung ${ }^{4}$, Detlef Lohse ${ }^{1,5}$ \\ and Sander G. Huisman ${ }^{1, \dagger}$ \\ ${ }^{1}$ Physics of Fluids Group, Max Planck UT Center for Complex Fluid Dynamics, MESA+ Institute and \\ J.M. Burgers Centre for Fluid Dynamics, University of Twente, P.O. Box 217, 7500 AE Enschede, \\ The Netherlands \\ ${ }^{2}$ Soft Matter, Fluidics and Interfaces, MESA+ Research Institute, University of Twente, P.O. Box 217, \\ 7500AE Enschede, The Netherlands \\ ${ }^{3}$ Institut für Strömungsmechanik und Aerodynamik, Universität der Bundeswehr München, \\ Werner-Heisenberg-Weg 39, 85577 Neubiberg, Germany \\ ${ }^{4}$ Department of Mechanical Engineering, University of Melbourne, Melbourne, Victoria 3010, Australia \\ ${ }^{5}$ Max Planck Institute for Dynamics and Self-Organization, Am Faßberg 17, 37077 Göttingen, Germany
}

(Received 11 September 2020; revised 21 February 2021; accepted 13 April 2021)

Wall roughness induces extra drag in wall-bounded turbulent flows. Mapping any given roughness geometry to its fluid dynamic behaviour has been hampered by the lack of accurate and direct measurements of skin-friction drag. Here, the Taylor-Couette (TC) system provides an opportunity as it is a closed system and allows direct and reliable measurement of the skin-friction. However, the wall curvature potentially complicates the connection between the wall friction and the wall roughness. Here, we investigate a highly turbulent TC flow, with a hydrodynamically fully rough, rotating inner cylinder, while the outer cylinder is kept smooth and stationary. We carry out particle image velocimetry (PIV) measurements in the Twente Turbulent Taylor-Couette (T3C) facility with Reynolds numbers in the range of $4.6 \times 10^{5}<R e_{i}<1.77 \times 10^{6}$. From these we find, while taking into account the influence of the curved walls on the turbulence, that the observed effects of a hydrodynamically fully rough surface are similar for TC turbulence and flat-plate turbulent boundary layer flows (BL). Hence, the equivalent sand grain height $k_{s}$, that characterizes the drag properties of a rough surface, is similar for both flow geometries. Next, we obtain the dependence of the torque (skin-friction drag) on the Reynolds number for a given wall roughness, characterized by $k_{s}$, and find agreement with the same results derived from PIV measurements within $5 \%$. Our findings demonstrate

$†$ Email address for correspondence: s.g.huisman@utwente.nl

(C) The Author(s), 2021. Published by Cambridge University Press. This is an Open Access article, distributed under the terms of the Creative Commons Attribution licence (http://creativecommons.org/ licenses/by/4.0/), which permits unrestricted re-use, distribution, and reproduction in any medium, provided the original work is properly cited. 


\section{P. Berghout and others}

that global torque measurements in the TC facility could be well suited to reliably deduce wall-drag properties for any rough surface.

Key words: Taylor-Couette flow, turbulence modelling

\section{Introduction}

\subsection{Turbulent boundary layers over fully rough walls}

The transport of a fluid over a solid body or the transport of a solid body through a fluid is always hindered by friction forces acting on the interface between the solid and the fluid. Ideally, the solid surface is smooth, and the drag force is a purely viscous force. In nature and engineering applications, however, solid surfaces are nearly always rough. This means that, in addition to a modified viscous force, the roughness also results in a pressure contribution to the drag force ('pressure drag'), and consequently, an increase in the total drag force (the so-called 'drag penalty'). The contribution of the pressure drag to the total friction drag at the surface grows with increasing roughness height. Ultimately, when the pressure drag dominates, the surface is called hydrodynamically fully rough.

Owing to the obvious interest in reducing the drag penalty, substantial research has been carried out to investigate the effects of rough surfaces on wall-bounded turbulent flows (Jiménez 2004; Flack \& Schultz 2010; Chung et al. 2021). The key effect thereof is a downward shift (by $\Delta u^{+}$) of the mean streamwise velocity $\left(u^{+}\right)$in the overlap (or logarithmic) region of the turbulent boundary layer (BL) (Clauser 1954; Hama 1954). This shift can be considered as a direct measure of the drag penalty. The mean velocity profile for a rough wall in the overlap region is given by the Prandtl-von Kármán profile for smooth walls, minus this shift (Pope 2000)

$$
u^{+}=\frac{1}{\kappa} \log y^{+}+A-\Delta u^{+},
$$

where $y^{+}$is the wall-normal distance, and the von Kármán constant $\kappa \approx 0.40$ and $A \approx$ 5.0 are extracted from experimental or numerical data. The superscript ' + ' indicates a normalization of the velocity $u$ with the viscous velocity scale $u_{\tau}=\sqrt{\tau_{w} / \rho}$ and of the wall-normal distance $y$ with the viscous length scale $\delta_{v}=v / u_{\tau}$, where $\tau_{w}$ is the wall shear stress, $\rho$ is the fluid density and $v$ is the kinematic viscosity. For a fully rough surface, it can be derived from dimensional arguments that the velocity shift $\Delta u^{+}$depends logarithmically on the roughness height $k^{+}$, see e.g. Raupach, Antonia \& Rajagopalan (1991) and Pope (2000). The so-called fully rough asymptote of the roughness function is given by

$$
\Delta u^{+}=\frac{1}{\kappa} \log k_{s}^{+}+A-B
$$

where $B \approx 8.5$ is the Nikuradse constant. The equivalent sand grain roughness height $k_{s}^{+}$ is obtained by fitting, such that the velocity shift of any fully rough surface collapses with the velocity shift of sand grains in turbulent pipe flow, which has historically grown to be the reference case (Nikuradse 1933). Hence, the key objective in research of wall bounded turbulent flows over rough surfaces is to relate the statistics of a rough surface to the value of $k_{S}$, which characterizes the roughness (Forooghi et al. 2017). 


\section{Taylor-Couette flow with rough surfaces}

\subsection{Taylor-Couette flow}

TC flow - the flow between two coaxial, independently, rotating cylinders - is a canonical system in turbulence (Taylor 1923; Grossmann, Lohse \& Sun 2016). Because the domain is closed in all directions, global balances can be derived and monitored, which enables extensive comparison among theory, experiments and simulations. Moreover, the torque (corresponding to the skin friction) can be measured accurately and directly (van Gils et al. 2012; Huisman et al. 2014), in contrast to measurements of skin friction in open systems.

The forcing strength of the system is quantified by the ratio of the centrifugal force and the viscous force, i.e. the Taylor number

$$
T a=\frac{1}{4}\left(\frac{1+\eta}{2 \sqrt{\eta}}\right)^{4} \frac{\left(r_{o}-r_{i}\right)^{2}\left(r_{i}+r_{o}\right)^{2}\left(\omega_{i}-\omega_{o}\right)^{2}}{v^{2}} .
$$

Here, $\eta$ is the geometric measure of curvature, namely the ratio $r_{i} / r_{o}$ of the radii of the cylinders. The subscripts $i$ and $o$ indicate inner cylinder and outer cylinder, respectively. The angular velocity is denoted by $\omega$, and $v$ is the kinematic viscosity.

The global response of the system is expressed as the Nusselt number $N u_{\omega}$, which is the ratio between the angular velocity flux $J^{\omega}$ in the radial direction and its laminar counterpart $J_{\text {lam }}^{\omega}$ (Eckhardt, Grossmann \& Lohse 2007), as

$$
N u_{\omega}=\frac{J^{\omega}}{J_{\text {lam }}^{\omega}}=\frac{r^{3}\left(\left\langle u_{r} \omega\right\rangle_{A(r), t}-v \partial_{r}\langle\omega\rangle_{A(r), t}\right)}{2 v r_{i}^{2} r_{o}^{2}\left(\omega_{i}-\omega_{o}\right) /\left(r_{o}^{2}-r_{i}^{2}\right)} .
$$

Here, $\langle\cdot\rangle_{A(r), t}$ denotes averaging over the cylinder surface $A(r)$ and over time $t$. The Nusselt number $N u_{\omega}$ is related to the torque $\mathcal{T}$ required to drive the inner cylinder. In non-dimensional form, the torque can be expressed as

$$
G=\frac{\mathcal{T}}{2 \pi L \rho v^{2}}=N u_{\omega} \frac{J_{\text {lam }}^{\omega}}{v^{2}}
$$

From here on, we assume inner cylinder rotation only, hence $\omega_{o}=0$, as this corresponds to our experiments where we kept the (smooth) outer cylinder stationary at all times.

The torque is directly related to the wall shear stress $\tau_{w}=\mathcal{T} /\left(2 \pi r_{i}^{2} L\right)$. As commonly used in other canonical systems (e.g. the flat-plate BL), we define the friction factor $C_{f}$ following Cheng, Pullin \& Samtaney (2020)

$$
C_{f}=\frac{8 R e_{\tau}^{2}}{R e_{i}^{2}}=\frac{4 N u_{\omega}}{\eta(1+\eta) R e_{i}}
$$

where $\operatorname{Re}_{i}=r_{i} \omega_{i} d / v, \operatorname{Re}_{\tau}=u_{\tau} d /(2 v)$ and $d=r_{o}-r_{i}$.

The turbulent flow in the TC set-up is strongly influenced by the curvature of its bounding walls, i.e. the cylinders that drive the flow. This distinguishes turbulent TC flow from turbulent flows in other canonical systems. Bradshaw (1969) realized that the effects of curvature on a turbulent BL are very similar to the effects of buoyancy stratification on a turbulent BL (Obukhov 1971). In analogy to the Obukhov length (Obukhov 1971; Monin \& Yaglom 1975), he derived a length scale that separates the curved BL in a region 


\section{P. Berghout and others}

where the effects of shear dominate (i.e. production of turbulence is dominated by shear production), and a region further away from the wall where curvature effects dominate (i.e. the production of turbulence is dominated by curvature). For smooth wall TC turbulence, this 'curvature Obukhov length' is well approximated by Berghout et al. (2020)

$$
L_{c, s}=\frac{u_{\tau}}{\kappa \omega_{i}},
$$

where shear dominates for $y \lesssim 0.20 L_{c, s}$, shear and curvature effects are both significant for $0.20 L_{c, s} \lesssim y \lesssim 0.65 L_{c, s}$, and curvature effects dominate at $0.65 L_{c, s} \lesssim y$. Here, $y$ is the distance to the cylinder, which is $y=r-r_{i}$ for the inner cylinder BL and $y=r_{o}-r$ for the outer cylinder BL. For the derivation of $L_{c}^{+}$, we refer to $\S 3.1$. Using data of the mean velocity profiles from PIV in turbulent TC flow (Huisman et al. 2013; van der Veen et al. 2016) and direct numerical simulations (DNS) (Ostilla-Mónico et al. 2015), the mean angular logarithmic velocity profile in the region of the turbulent BL, where curvature effects are important, was recently obtained for smooth wall TC flow (Berghout et al. 2020). By employing a matching argument between the velocity profiles of the turbulent BL and the bulk region, following the work of Cheng et al. (2020), an analytical expression for $N u(T a)$ was derived (Berghout et al. 2020).

The effects of irregular boundaries (extended transverse bars in the 'obstacle regime', as referred to by Jiménez (2004)) on turbulent TC flow were previously investigated by means of experiments (Cadot et al. 1997; van den Berg et al. 2003; Verschoof et al. 2018; Zhu et al. 2018) and DNS (Zhu, Verzicco \& Lohse 2017; Zhu et al. 2018). The ratio $k / d$ between the height of the bars and the gap width $d=r_{o}-r_{i}$ was as large as $k / d=0.05$ or even 0.1. Later, Berghout et al. (2019) numerically studied the effects of sand grain roughness $(k / d=0.019-0.087)$ on the turbulent TC velocity profiles, and found similar transitionally rough behaviour as the sand grain roughness of Nikuradse (1933) in turbulent pipe flow. However, we note that both the experimental and computational studies in TC flow suffered from a limited scale separation between the roughness scale $k$ and the gap width $d$.

In this paper, we study the effects of a hydrodynamically fully rough inner cylinder on the turbulent wall-bounded flow, with small roughness $k / d=0.014$, where $k \equiv 6 k_{\sigma}$, and $k_{\sigma}$ is the standard deviation of the roughness elevation, following Squire et al. (2016). In particular, we keep $k$ much smaller than the curvature Obukhov length $L_{c}$ (see (3.2)), namely $k / L_{c}=0.078-0.090$. We will demonstrate that to study the effects of roughness on a turbulent flow in TC, $k \ll d$ is not enough. Rather, $k \ll L_{c}$ must also hold to ensure that effects related to the streamwise curved geometry are not influencing the effects of the roughness.

Hence, we hypothesize that effects of roughness in TC turbulence (where $k \ll L_{c}$ ) are similar to the effects of roughness in other canonical systems without streamwise curvature. Thus, global measurements in the (closed) TC facility can be employed to characterize drag properties of the rough surface. The outer cylinder remains smooth to allow for optical access of the velocity profiles.

The paper is organised as follows: In $\S 2$, we describe the experimental methods. We then $(\S 3)$ discuss the relevant dynamical length scales in the experiment, and elaborate on the different regions in the BL where turbulent production is dominated by shear effects and where effects related to the streamwise curvature of the set-up play a role. We also comment on the scale separation and show that the roughness mainly affects the inertial shear dominated regime, and hence, effects from the streamwise curved geometry of the TC flow do not modify the velocity shift. In $\S 4$, we use the mean velocity profiles of the inner cylinder boundary layer to show that, apart from the shift, the velocity profiles for rough and smooth inner cylinders are the same. We use this in $\S 5$ to 


\section{Taylor-Couette flow with rough surfaces}

calculate the angular velocity shift $\Delta \omega^{+}$, from which the equivalent sand grain roughness height is determined in $\S 6$. In $\S 7$, we demonstrate that the bulk region of the flow is of constant angular momentum, which is used in $\S 8$ to obtain a relation between the torque (skin-friction drag) and the Reynolds number for given surface roughness $k_{s}$, in agreement with our experimental results. The paper ends with a summary, conclusions and an outlook $(\S 10)$.

\section{Experimental set-up and methods}

\subsection{Experimental set-up}

The experiments were performed in the Twente Turbulent Taylor-Couette $\left(\mathrm{T}^{3} \mathrm{C}\right)$ facility (van Gils et al. 2011a), with water as the working fluid. We used a fully rough inner cylinder with an outer radius of $r_{i}=201.2 \mathrm{~mm}$, which consisted of a smooth inner cylinder with radius $200.0 \mathrm{~mm}$ and a support layer of the roughness with a thickness of $1.2 \mathrm{~mm}$, and a transparent outer cylinder with an inner radius of $r_{o}=279.4 \mathrm{~mm}$. This gave a radius ratio of $\eta=r_{i} / r_{o}=0.720$ and a gap width $d=r_{o}-r_{i}=78.2 \mathrm{~mm}$ for the rough wall cases. For the smooth wall calculations in $\S 8$, we used $\eta=0.714$. The cylinders had a height of $L=927 \mathrm{~mm}$ and an aspect ratio of $\Gamma=L / d=11.9$. For inner cylinder rotation only (the outer cylinder is stationary), the Reynolds number, $R e_{i}$, is defined with the velocity of the inner cylinder, $r_{i} \omega_{i}$, and the gap width, $d$, as

$$
\operatorname{Re}_{i}=\frac{\omega_{i} r_{i} d}{v}
$$

Using the viscous velocity, $u_{\tau}$, which is obtained from torque measurements, the friction Reynolds number, $R e_{\tau}$, is defined as

$$
\operatorname{Re}_{\tau}=\frac{u_{\tau}(d / 2)}{v} .
$$

The roughness used was P36 grit sandpaper (VSM, ceramic industrial grade), which was fixed to the entire surface of the inner cylinder using double-sided adhesive tape (tesa 51970). We define the characteristic length scale of the roughness as $k \equiv 6 k_{\sigma} \approx 1.07 \mathrm{~mm}$ (corresponding to the $99.8 \%$ interval of the height), where $k_{\sigma}$ is the standard deviation of the local roughness height $h(x, y)$ (quantified using confocal microscopy (Bakhuis et al. 2020) on a square sample with sides of $25 \mathrm{~mm}$ from the rough sandpaper), and $k / d=$ 0.014. For more details of the roughness height properties, see table 1 of Bakhuis et al. (2020).

\subsection{Experimental procedure}

We performed seven experiments with different rotation rates of the inner cylinder, see table 1. During all these experiments, the torque $\mathcal{T}$ that is required to drive the inner cylinder at fixed rotational velocity was measured. The hollow reaction torque sensor that connects the drive shaft to the middle section of the inner cylinder is indicated in figure 1(a). By only measuring the torque on the middle section, possible end-plate effects were eliminated (van Gils et al. 2012). During the torque measurements, PIV was used to obtain the velocity field in the gap. To quantify the reproducibility of our torque measurements, we compared the torque data that were captured during the PIV experiments with three separate torque measurements thereafter. We found a spread in $\mathcal{T}$ of smaller than $4 \%$ for all cases. These direct and reproducible measurements of the torque 


$\begin{array}{lccccccccc}T a\left(\times 10^{12}\right) & R e_{i}\left(\times 10^{6}\right) & N u_{\omega} & C_{f}\left(\times 10^{-3}\right) & R e_{\tau}\left(\times 10^{3}\right) & L_{c}^{+}\left(\times 10^{3}\right) & k_{\sigma}^{+} & k_{s}^{+} & \Delta \omega^{+} & u_{\tau}\left(\mathrm{m} \mathrm{s}^{-1}\right) \\ 0.31 & 0.46 & 312 & 2.21 & 7.6 & 2.6 & 34 & 189 & 9.31 & 0.21 \\ 0.57 & 0.62 & 403 & 2.13 & 10.0 & 3.3 & 44 & 245 & 10.09 & 0.26 \\ 0.92 & 0.78 & 513 & 2.14 & 12.7 & 4.1 & 57 & 317 & 10.80 & 0.33 \\ 1.47 & 0.99 & 643 & 2.12 & 16.0 & 5.0 & 72 & 400 & 11.46 & 0.41 \\ 2.18 & 1.20 & 784 & 2.12 & 19.5 & 6.0 & 87 & 484 & 12.06 & 0.50 \\ 3.55 & 1.54 & 998 & 2.11 & 24.9 & 7.6 & 111 & 617 & 12.80 & 0.61 \\ 4.71 & 1.77 & 1137 & 2.09 & 28.5 & 8.5 & 127 & 706 & 13.21 & 0.68 \\ 6.15 & 2.00 & 653 & 1.07 & 23.1 & 6.9 & 0 & - & - & 0.58\end{array}$

Table 1. Control parameters, global response and relevant length scales, measured during the PIV measurements. The $T a$ or $R e_{i}$ characterize the driving of the system. Here, $N u_{\omega}$ is the dimensionless angular velocity flux, $C_{f}$ is the friction factor, $L_{c}^{+}$is the curvature Obukhov length as defined in (3.2) and $k_{\sigma}^{+}$ is the standard deviation of the sandpaper roughness $k_{\sigma}^{+}=\sqrt{\left\langle\left(h_{r}-\left\langle h_{r}\right\rangle\right)^{2}\right\rangle} / \delta_{v}$, where $\langle\cdot\rangle$ refers to spatial averaging and $h_{r}$ is the mean roughness elevation, see Bakhuis et al. (2020). The equivalent sandgrain height is $k_{s}^{+}=5.56 k_{\sigma}^{+}$. The velocity shift is $\Delta \omega^{+}$and $u_{\tau}$ is the friction velocity. The bottom row presents the values corresponding to the smooth wall measurement of Huisman et al. (2013).

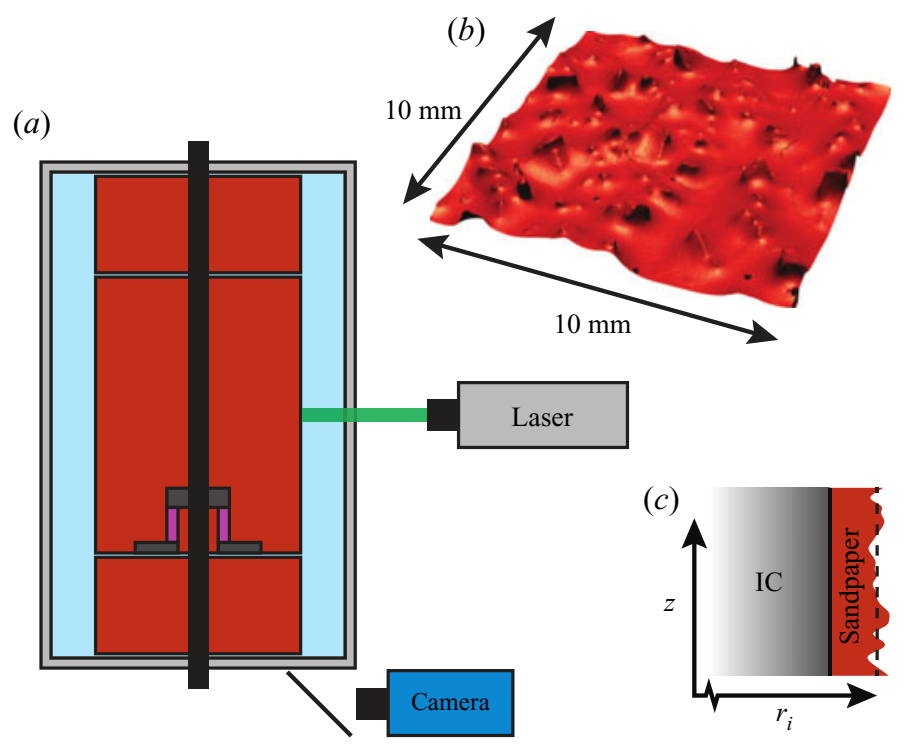

Figure 1. (a) Cross-section of the TC geometry. The tracer particles are illuminated by a $1 \mathrm{~mm}$ thick horizontal laser light-sheet from the right at mid-height of the cylinders. The light scattered by the tracers is imaged from the bottom through a mirror. The torque sensor only measures the torque of the middle part of the inner cylinder. (b) Three-dimensional visualization of the confocal scan of the used sandpaper. (c) Cross-section of the inner cylinder with the sandpaper attached to the surface.

(friction) have an accuracy that is comparable to the measurement accuracy of wall shear stress in flat-plate BLs, by means of a drag balance (Baars et al. 2016).

For the PIV measurements, fluorescent polymer tracer particles (Dantec FPP-RhB-10 1-20 $\mu \mathrm{m}$ ) were added to the working fluid. A horizontal laser sheet with a thickness of approximately $1 \mathrm{~mm}$ illuminated the tracer particles in the working liquid at mid-height, through the transparent outer cylinder. The laser sheet was created using a frequency doubled Quantel EverGreen $200 \mathrm{~mJ}$ laser. The fluorescent light emerging from the tracer 


\section{Taylor-Couette flow with rough surfaces}

particles was imaged from below, through a window in the bottom plate of the apparatus. For this, a $45^{\circ}$ mirror was positioned under the bottom plate as drawn schematically in figure 1. The camera was a high-resolution sCMOS camera (LaVision PCO.edge), with a resolution of $2560 \mathrm{px} \times 2160 \mathrm{px}$ and a pixel size of $6.5 \mu \mathrm{m}$. A $100 \mathrm{~mm}$ focal length objective (Zeiss Makro Planar, $100 \mathrm{~mm}$ ) was used, which gave an optical magnification of 0.17 .

For each rotational velocity of the inner cylinder, $10^{4}$ image pairs were acquired at a recording frequency equal to the rotation rate. The mean velocity distribution in the horizontal plane was computed using single-pixel ensemble correlation (Kähler, Scholz \& Ortmanns 2006; Kähler, Scharnowski \& Cierpka 2012). The spatial resolution was $50 \mu \mathrm{m}$, which led to approximately 1600 independent measurement points in the radial direction, evenly spread over the entire gap. From the correlation function (obtained for every pixel), one can directly extract the standard deviation of the velocity by integrating the probability density function $\sigma(u)=\int_{-\infty}^{\infty}(u-\langle u\rangle)^{2} \operatorname{PDF}(u) \mathrm{d} u$, see Scharnowski, Hain \& Kähler (2012). This ensures that all turbulent scales are included in the standard deviation, as opposed to regular PIV analysis. The velocity profiles were smoothed using a Gaussian filter with a standard deviation of $\sigma \approx 0.5 \mathrm{~mm}$.

\section{Curvature effects, the mean velocity profile and scale separation}

\subsection{The relative effects of curvature and shear}

To characterize and quantify the relative effects of shear and curvature in TC turbulence, we studied the ratio $S^{-1}$, which is the ratio of turbulence production by shear over turbulence production by curvature (Bradshaw 1969; Townsend 1976; Berghout et al. 2020)

$$
S^{-1}=\frac{\overline{u_{\theta}^{\prime} u_{r}^{\prime}} \frac{\mathrm{d}}{\mathrm{d} r} U}{\frac{1}{r} \overline{u_{\theta}^{\prime} u_{r}^{\prime}} U}=\frac{1}{\omega} \frac{\mathrm{d} U}{\mathrm{~d} r},
$$

where $u_{\theta}^{\prime}$ and $u_{r}^{\prime}$ are the azimuthal and radial velocity fluctuations, respectively, and $\overline{u_{\theta}^{\prime} u_{r}^{\prime}}$ is the Reynolds stress. The mean azimuthal velocity is denoted by $U$ and $\omega=U / r$ is the mean angular velocity. The curvature Obukhov length $L_{c}$ defined in (1.7) for a smooth wall marks the transition from a region where the production of turbulence is dominated by shear $\left(y<0.20 L_{c}\right)$ to a region where it is affected by curvature $\left(y>0.20 L_{c}\right)$. The definition from (1.7) builds on the existence of a shear logarithmic region, where the gradient of the mean angular velocity is $(\mathrm{d} /(\mathrm{d} r)) U=u_{\tau} /(\kappa y)$. Once we approximate the mean angular velocity with $\omega=\omega_{i}$, we find that for $S^{-1}=\left(u_{\tau} /\left(\kappa \omega_{i} y\right)\right)=1$, we have $y=L_{c}$. Physically this means that at a distance of $L_{c}$ from the wall, the shear and curvature production of turbulence are of equal magnitude. The angular velocity scale for rough walls is approximated as $\omega=\omega_{i}+\Delta \omega$. Thus, the generic curvature Obukhov length $L_{c}$ for smooth and rough walls can be defined with the inner cylinder rotation rate $\omega_{i}$ and the wall-shear stress $\tau_{w}$ only, similar to (1.7), but now for a rough wall,

$$
L_{c}=\frac{u_{\tau}}{\kappa\left(\omega_{i}+\Delta \omega\right)},
$$

so that $L_{c}^{+}\left(\Delta \omega^{+}=0\right)=L_{c, s}^{+}$.

Figure 2(a) presents the compensated gradient of the mean angular velocity profile versus $S^{-1}$, calculated from the PIV results. We find fair collapse of the velocity gradients 


\section{P. Berghout and others}
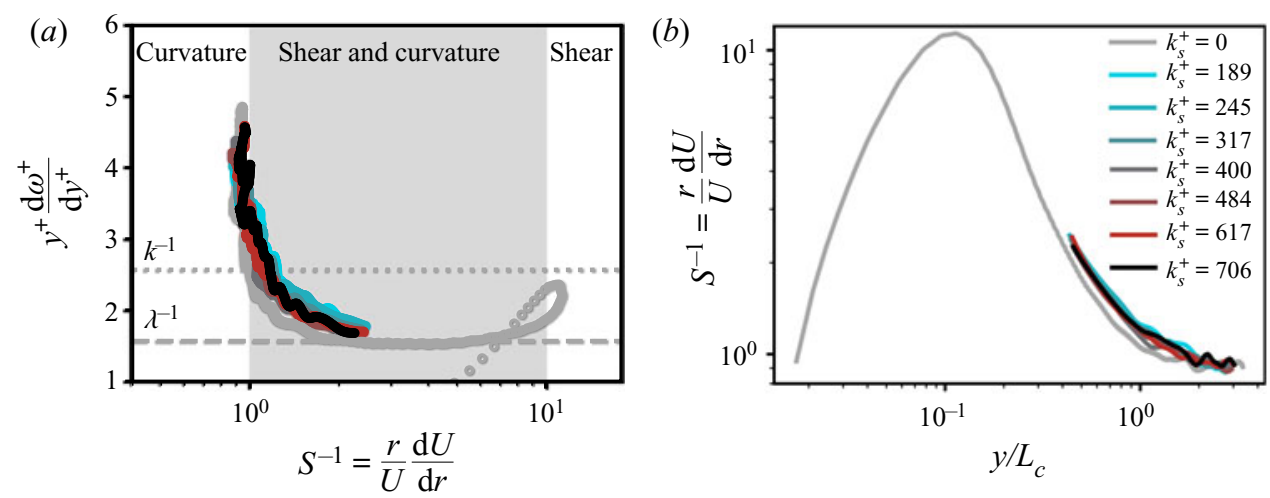

Figure 2. (a) Compensated gradient of the mean angular velocity profile versus the ratio $S^{-1}$ between the turbulence production by shear and that by curvature, see (3.1). Dotted and dashed lines represent the slope of the logarithmic velocity profile of the shear and the curvature dominated regimes, $\kappa^{-1}$ and $\lambda^{-1}$, respectively. ( $b$ ) Ratio $S^{-1}$ versus the wall-normal distance shifted with the wall offset, $y / L_{c}=\left(r-r_{i}-2 k_{\sigma}\right) / L_{c}$, where $2 k_{\sigma}$ is the approximated wall offset of the sandpaper. Coloured lines are calculated from the PIV data of the rough wall cases. The grey line $\left(k_{\sigma}^{+}=0\right)$ is the smooth wall profile at $T a=6.2 \times 10^{12}\left(\operatorname{Re}_{\tau}=23093\right)$, obtained from Huisman et al. (2013).

of the smooth (grey) and rough (coloured) wall profiles. When the effects of curvature are negligible $S^{-1} \geq O(10)$, the compensated gradient of the velocity profile approaches $\kappa^{-1} \approx 2.5$. This occurs in a very small region close to the wall, where we cannot measure owing to the presence of the sandpaper roughness. For the rough and smooth wall velocity profiles, we find that the compensated gradient approaches $\lambda^{-1}$ in the region where curvature and shear affect the flow. For $S^{-1} \leq 1$, curvature effects dominate the flow, and a constant angular momentum region (i.e. the bulk flow) is established (Berghout $e t$ al. 2020).

\subsection{The mean angular velocity profile}

Figure 2(a) shows that the curvature- and shear-affected region of the BL contains a constant compensated gradient $\left(=\lambda^{-1}\right)$ of the mean angular velocity. From this observation, Berghout et al. (2020) obtained an equation of the mean angular velocity in the shear- and curvature-affected region in the BL (in short 'curvature log').

The offset of the logarithmic velocity profile (with slope $\lambda^{-1}$ ) in the curvatureand shear-affected region, as indicated in figure 3, is a function of the wall normal location where curvature-related effects impact the flow. From PIV results, the exact location was found to be $y^{+}=0.20 L_{c}^{+}$, with $L_{c}^{+}$defined in (3.2). Therefore, the offset is $\kappa^{-1} \log 0.2 L_{c}^{+}+A$, where $A=5.0$ is the offset of the logarithmic velocity profile in the shear-affected region (Pope 2000). The transition in the logarithmic velocity profile from the shear-affected region to the curvature- and shear-affected region at $y^{+}=0.20 L_{c}^{+}$is not sharp but gradual. To account for this, we introduce a constant $C_{b l}$ that connects the logarithmic velocity profiles of both regions. Berghout et al. (2020) found that $A+C_{b l}+$ $(1 / \kappa-1 / \lambda) \log (0.2)=1.0$ for the inner cylinder, and the mean angular velocity equation above $y^{+}=0.20 L_{c}^{+}$as

$$
\omega^{+}=\frac{1}{\kappa} \log 0.2 L_{c}^{+}+A+C_{b l}+\frac{1}{\lambda} \log \frac{y^{+}}{0.2 L_{c}^{+}}=\frac{1}{\lambda} \log y^{+}+\left(\frac{1}{\kappa}-\frac{1}{\lambda}\right) \log L_{c}^{+}+1.0,
$$




\section{Taylor-Couette flow with rough surfaces}

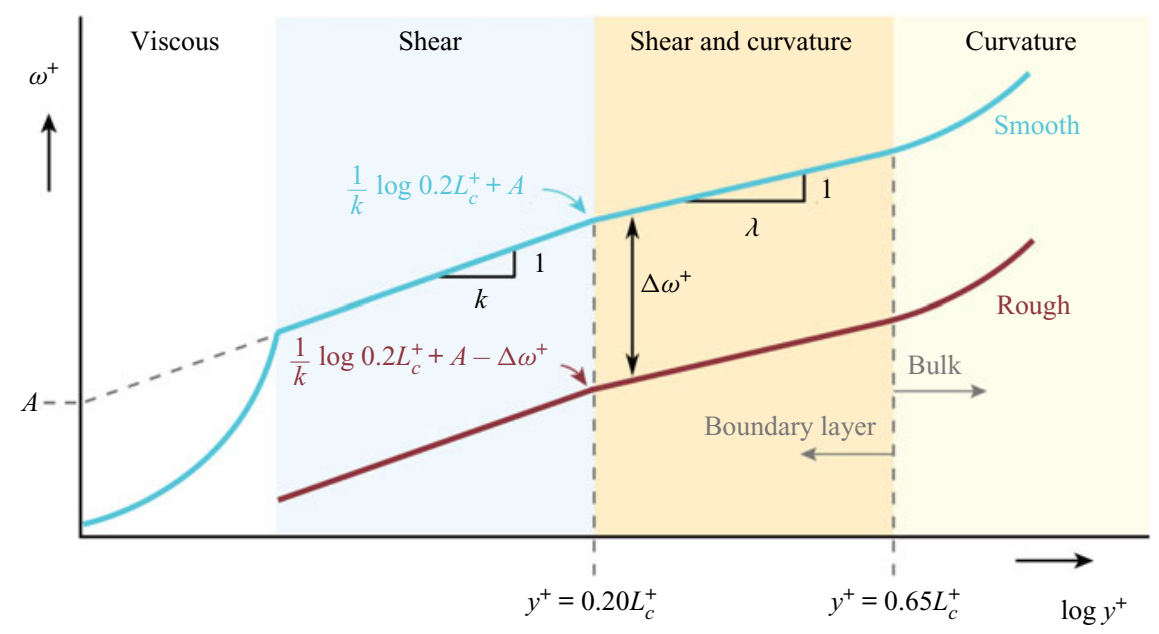

Figure 3. Schematic of the various regions in smooth and IC-rough turbulent TC flows at matched $L_{c}^{+}$. The height $y^{+}=0.65 L_{c}^{+}$is defined as the location where the logarithmic profile with slope $\lambda^{-1}$ ends and the constant angular momentum region of the bulk velocity starts.

with $C_{b l}=-3.30$. The transition from the curvature- and shear-affected region to the constant angular momentum region occurs at $y^{+}=0.65 L_{c}^{+}$. We take this height as our definition of the boundary layer height, above which is the bulk region of constant angular momentum.

\subsection{Scale separation}

Key to the understanding of the effects of roughness in TC turbulence is the concept of scale separation. To illustrate this, in figure $2(b)$, we plot $S^{-1}$ versus the wall-normal distance $y / L_{c}=\left(r-r_{i}-2 k_{\sigma}\right) / L_{c}$. We note that the wall offset (or zero plane displacement) $2 k_{\sigma}^{+}$of the rough wall is an approximation. Because we focus on the flow region that is relatively far away from the wall, and $k_{s} / d \ll 1$, the choice of the zero plane displacement $h_{v}$ has negligible influence on the results, as far as $0<h_{v}<k$ (Raupach et al. 1991; Squire et al. 2016). To quantify this, we set $h_{v}=0$ and $h_{0}=6 k_{\sigma}$ (valley and peak of the roughness) and find that $\Delta \omega^{+}$only varies by $4 \%$. As a reference, we also plot the smooth wall profile (grey) at $T a=6.2 \times 10^{12}$ (Huisman et al. 2013), together with the rough wall profiles (colours).

Table 1 presents the relevant dynamical length scales in the experiments: namely, $R e_{\tau}$, $L_{c}^{+}$and $k_{\sigma}^{+}$. The friction Reynolds number $R e_{\tau}$ from (2.2) gives the ratio of the largest dynamical length scale in the TC set-up to the viscous length scale $\delta_{v}$. Here, $R e_{\tau}$ is of the same order as in the smooth wall TC experiments by Huisman et al. (2013), where it was $R e_{\tau}=488-23093$, which is comparable to the rough BL experiments by Squire et al. (2016), where $\operatorname{Re}_{\tau}=2890-29900$.

The roughness scale in our experiments is much larger than the viscous length scale $\delta_{v}$, i.e. $k_{\sigma}^{+}=34-127 \gg 1\left(k_{s}^{+}=188-704 \gg 1\right)$, and thus pressure drag dominates over viscous drag. For the flat-plate BL experiments of Squire et al. (2016) in the fully rough regime, we estimate that $k_{\sigma}^{+}=9-12$ is required, based on the data for which $\Delta U^{+}>8.0$ in Squire et al. (2016). Hence, we are confident that we are indeed far in the fully rough regime. We also find that the roughness sublayer height $\approx(6-9) k_{\sigma}^{+}$(approximately 0.5 times the roughness spacing Chung et al. 2021) is smaller than the outer bound 


\section{P. Berghout and others}

of the shear dominated logarithmic region, $\approx 0.2 L_{c}^{+}$. For the lowest roughness, we have $9 k_{\sigma}^{+} / 0.2 L_{c}^{+}=0.60$, and for the highest roughness, it is $9 k_{\sigma}^{+} / 0.2 L_{c}^{+}=0.66$. This separation of length scales allows for a region where the logarithmic velocity profile can form, albeit marginally. For example, in the smooth wall experiments of Huisman et al. (2013), such a profile was found between $50 \leq y^{+} \leq 600$ for comparable $T a$. We finally find that the outer bound of the curvature dominated logarithmic region $L_{c}^{+}$is smaller than the outer length scale $R e_{\tau}$, so that $0.65 L_{c}^{+} / R e_{\tau} \approx 0.33$. For $y^{+}>0.65 L_{c}^{+}$, the curvature dominated bulk, a constant angular momentum region forms. The occurrence and extent of this constant angular momentum region depends on the radius ratio $\eta$, i.e. $L_{c}^{+} / \operatorname{Re}_{\tau}$ depends on $\eta$.

From table 1 , we find that $\delta_{v} \ll k_{\sigma}<0.20 L_{c}$, hence the roughness only affects the inertial region where curvature effects are negligible. We therefore expect that the velocity shift of that region is similar to that of identical sandpaper in a flat-plate turbulent BL. Therefore, we would expect a fully rough asymptote with slope $\kappa^{-1}$ and a similar value of $k_{s}$ as we would measure for identical sandpaper in a flat-plate turbulent BL.

\section{Mean velocity profiles of the inner cylinder boundary layer}

In this paper, we will show the angular velocity profile $\omega^{+}\left(y^{+}\right)$rather than the azimuthal velocity profile $u^{+}\left(y^{+}\right)$, as it is $\omega^{+}\left(y^{+}\right)$that is expected, given the arguments based on the Navier-Stokes equations, to have a logarithmic profile (Grossmann, Lohse \& Sun 2014).

Figure 4(a) shows the angular velocity profiles over the rough wall $\omega^{+}=\left\langle\omega_{i}-\right.$ $\omega(r)\rangle_{t} / \omega_{\tau}$, with $\omega_{\tau}=u_{\tau} / r_{i}$, versus the wall-normal coordinate $y^{+}$. In this and the next section, we focus our analysis on the mean velocity profiles of the inner cylinder BL, hence $u_{\tau}=u_{\tau, i}$ throughout. In $\S 7$, we will report on the bulk profiles. We refer to Berghout et al. (2020) for an analysis of the smooth velocity profiles of the outer cylinder BL.

Figure 4(a) shows that with increasing viscous scaled roughness height, the rough wall profiles are increasingly shifted downwards, as expected. More importantly, we find from the diagnostic function $y^{+}\left(\mathrm{d} \omega^{+} / \mathrm{d} y^{+}\right)$(a useful representation of the compensated gradients (Pope 2000)) in figure 4(b) that the slope $\lambda^{-1}$ of the shear- and curvature-affected logarithmic region is the same for rough wall TC turbulence as for smooth wall TC turbulence (grey line). Unfortunately, we could not resolve the very thin spatial region where a shear-dominated logarithmic was found by Huisman et al. (2013), as the roughness peaks obstruct the view for the PIV very close to the wall. Note that the range of $y^{+} / L_{c}^{+}$in figure $4(b)$ is slightly shorter than in figure 5(a), because we employ a Gaussian smoothing before calculating the gradient of the velocity.

For a rough wall, $\Delta \omega^{+}$is a function of the equivalent sand grain roughness height $k_{s}^{+}$and the curvature length $L_{c}$, so that $\Delta \omega^{+}\left(k_{s}^{+}, k_{s} / L_{c}\right)$. When $k_{s} \ll L_{c}$, the angular velocity shift only depends on $k_{s}$, and the shift becomes $\Delta \omega^{+}\left(k_{s}^{+}\right)$. Because the inner cylinder rotates, the plus sign in the denominator of (3.2) is connected with the increase of angular fluid velocity in the inner cylinder BL owing to the roughness. When we normalize the wall-normal distance with $L_{c}^{+}$, we expect the transition from a curvature logarithmic velocity profile to the constant angular momentum bulk velocity profile to occur at $y^{+} / L_{c}^{+}=0.65$. In figure $4(b)$, we find a fair collapse of both smooth and rough wall profiles in the wall-normal direction, when normalized with curvature length $L_{c}^{+}$. The slopes of the curvature-affected logarithmic region of all profiles, rough and smooth, in the domain $0.20 L_{c}^{+}<y^{+}<0.65 L_{c}^{+}$, fall in the range $\lambda=0.60-0.65$. 
(a)

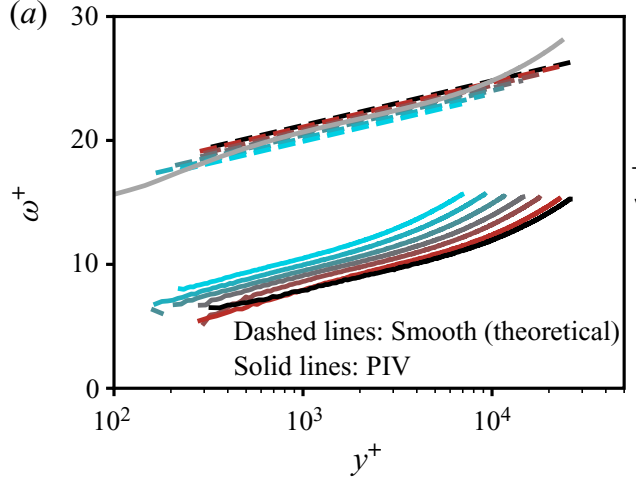

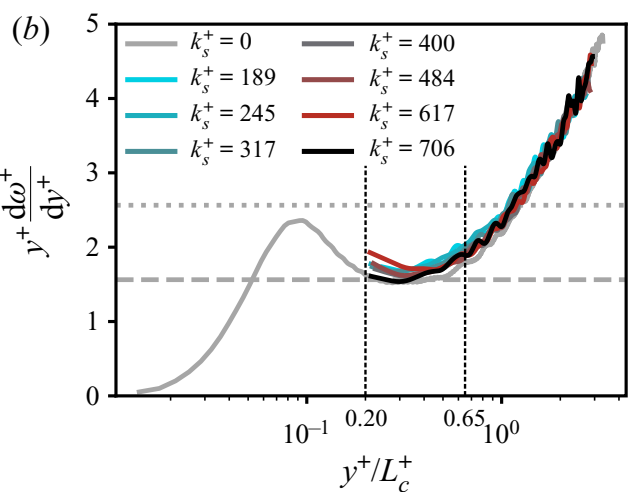

Figure 4. (a) Mean angular velocity $\omega^{+}$versus the wall-normal distance $y^{+}$. The solid lines are the measured rough wall profiles. The dashed lines represent the theoretical smooth wall reference profiles (colours are the same), calculated from (3.3) and at matching $L_{c}^{+}$and $R e_{\tau}$ with the rough wall profiles. (b) The compensated gradient of the rough wall profiles in $(a)$, where the wall-normal distance is normalized with the curvature length $L_{c}^{+}$. The colours are the same in both figures. The grey line is the smooth wall profile at $T a=6.2 \times 10^{12}$, obtained from Huisman et al. (2013). The dashed horizontal line represents the slope $\lambda^{-1}$ of the logarithmic velocity profile in the region where turbulence production is governed by effects of curvature and shear. The dotted horizontal line represents the slope $\kappa^{-1}$ of the logarithmic velocity profile in the region where turbulence production is dominated by shear.
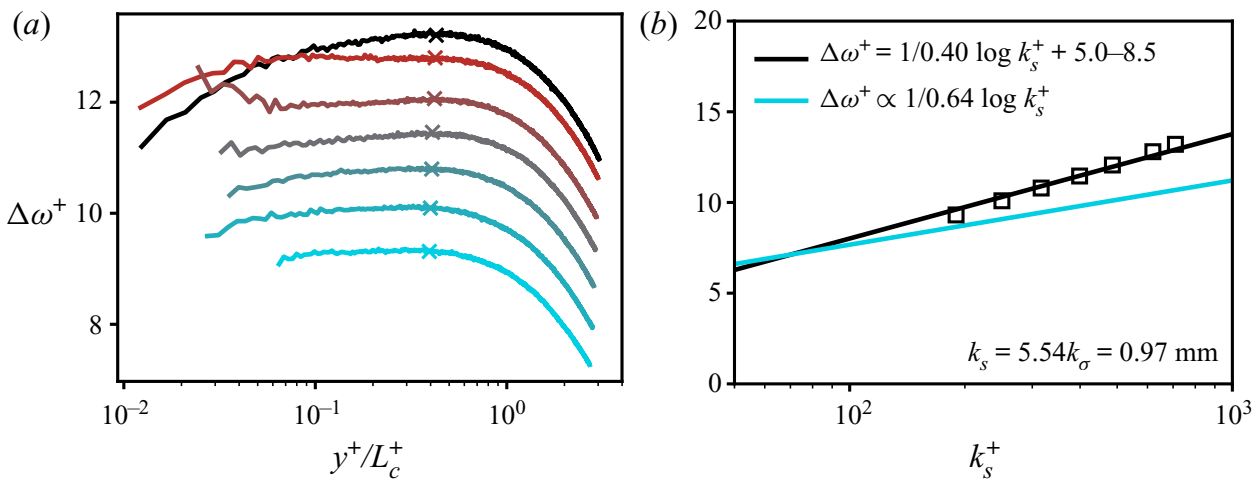

Figure 5. Velocity shift $\Delta \omega^{+}$of the rough wall profiles with respect to the reference smooth wall profiles. (a) Velocity shift versus the wall-normal distance $y^{+} / L_{c}^{+}$(colours are the same as figure 4). (b) The velocity shift $\Delta \omega^{+}$, crosses in $(a)$, versus the equivalent sand grain height $k_{s}^{+}$. Black symbols are the experimental values. The solid black line is the fully rough asymptote of Nikuradse (1933), (5.1). The solid blue line is an illustration of the curvature fully rough asymptote, with slope $\lambda^{-1}$ and arbitrary vertical shift.

\section{The fully rough asymptote}

From the observation that both smooth and rough wall velocity profiles possess the same slope $\lambda^{-1}$ of the curvature logarithmic region (figure $4 b$ ), we proceed to calculate the angular velocity shift $\Delta \omega^{+}$. The major advantage of the TC set-up is that the friction can be measured in a straightforward manner by measuring the torque. Hence, we can directly obtain the velocity shift by subtracting the rough velocity profile from the smooth velocity profile, rather than by means of fitting a velocity profile first to obtain $u_{\tau}$, e.g. the Clauser fit. Owing to the roughness, the angular velocity profiles in the shear logarithmic region are shifted, as discussed in $\$ 3.1$ and illustrated in figure 3 . This shift remains also 


\section{P. Berghout and others}

in the curvature logarithmic region, where we will now quantify it. The offset of that region scales with $(1 / \kappa) \log \left(0.20 L_{c}^{+}\right)+A$ (figure 3$)$. Hence, it is imperative to calculate the angular velocity shift from the smooth wall velocity profile at matching $L_{c}^{+}$.

Figure 4(a) shows these smooth wall profiles (dashed), where the colours match the respective rough wall cases, and both $L_{c}^{+}$and $R e_{\tau}$ are matched. The velocity shift $\Delta \omega^{+}\left(y^{+}\right)$from the theoretical smooth wall profile, (3.3), is plotted in figure 5(a). The horizontal plateaus confirm the similarity of the slopes of the velocity profiles. We extract $\Delta \omega^{+}$at $y^{+} \approx 0.4 L_{c}^{+}$and plot the shift versus the roughness height in figure $5(b)$. When we fit a function of the form $\Delta \omega^{+}=(1 / a) \log k^{+}+b$ through all seven data points, we obtain $a=0.34 \pm 0.02$ to within $13 \%$ of the von Kármán constant $\kappa=0.39$, as measured in TC turbulence (Huisman et al. 2013) as the slope of the shear dominated logarithmic profile. This confirms our hypothesis, as discussed in $\S 3.1$, where the fully rough asymptote for $\delta_{v} \ll k<L_{c}$ has slope $\kappa^{-1}$. For reference, this is much higher than $\lambda^{-1} \approx 0.64^{-1}$, blue line in figure $5(b)$.

To obtain a measure of the equivalent sand grain roughness height $k_{s}$, we fit the data points to the fully rough asymptote of Nikuradse (1933), with $\kappa \approx 0.40$, which is in accordance with the value reported for the fully rough asymptote (Jiménez 2004),

$$
\Delta \omega^{+}\left(k_{s}^{+}\right)=\frac{1}{\kappa} \log k_{s}^{+}+5.0-8.5,
$$

and obtain $k_{s}=5.54 k_{\sigma}=0.97 \mathrm{~mm}$. For reference, the typical grain size is estimated by $6 k_{\sigma}=1.05 \mathrm{~mm}$ (Bakhuis et al. 2020).

\section{The equivalent sand grain roughness height}

The hypothesis in this research, postulated in $\S 3.1$, is that the fully rough asymptote in TC turbulence with $\delta_{v} \ll k<L_{c}$ is the same (or very similar) to the fully rough asymptote in flows without streamwise curvature. We have already demonstrated in $\S 5$ that the slope $\kappa^{-1}$ of the fully rough asymptote is indeed (almost) the same. This leaves us with a comparison of the value of $k_{s}$ between TC turbulence and canonical systems without streamwise curvature.

In the literature, we have found two reports on turbulent flows over sandpaper roughness: the work of Squire et al. (2016), which employed 36 grit sandpaper in a turbulent BL, and Flack et al. (2007), who employed (12, 24, and 80) grit sandpaper in turbulent BL flow. In the rough wall TC experiments reported here, we used grit 36 sandpaper. However, it is essential to realize that sandpaper is not only defined by the grit size. Other statistics, such as the skewness (an important parameter (Forooghi et al. 2017), which is 0.93 here and only 0.09 in the paper by Squire et al. (2016)), do vary with manufacturing methods. We have tried to use the very same sandpaper type (SP40F, Awuko Abrasives) as Squire et al. (2016). Unfortunately, the sandpaper turned out not to be waterproof, and detached from the inner cylinder. We then applied new water-resistant sandpaper (VSM, P36 grit ceramic industrial grade), with different surface roughness statistics.

To compare the drag property of the sandpaper surfaces in TC to the respective sandpaper surfaces in literature, we plotted the relationship between $k_{s}$ and the root-mean-square height and skewness in figure 6 . The surface properties of the sandpaper surface from Flack et al. (2007) are taken from Flack \& Schultz (2010). The solid black line is the empirical correlation from Flack et al. (2020). We find that the relation between $k_{s}$ and the skewness $S k$ and the root-mean-square height $k_{\sigma}$ of the sandpaper used in our 


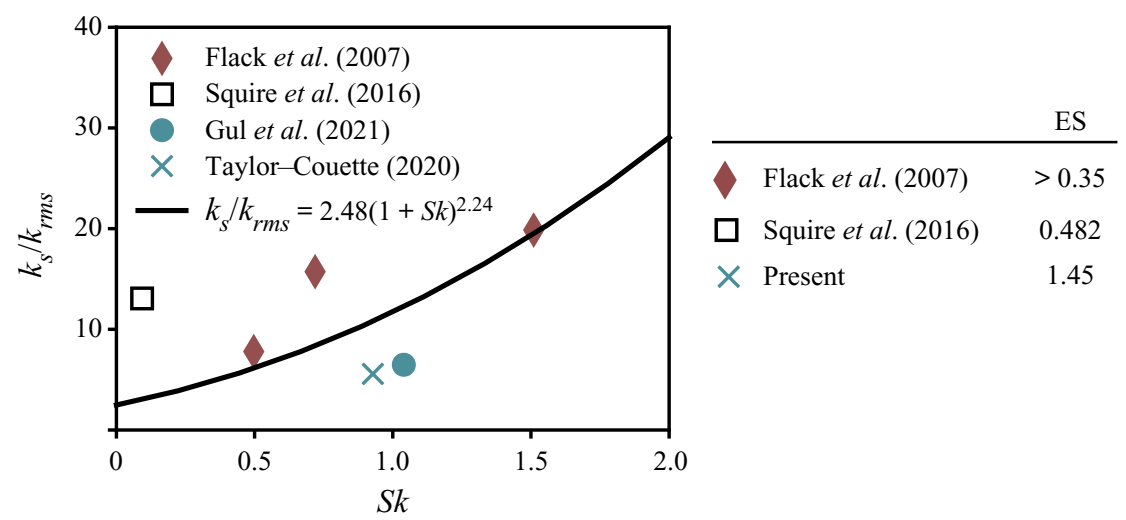

Figure 6. Relationship between the equivalent sand grain roughness height divided by the root-mean-square height $k_{s} / k_{\sigma}$, and the skewness parameter $S k$ of different sandpaper surfaces. The solid black line is the empirical correlation for $S k>0$ from Flack et al. (2020). Data from turbulent boundary layer flow using grit (12, 24 and 80) sandpaper (Flack, Schultz \& Connelly 2007), of which the surface statistics are listed by Flack \& Schultz (2010), turbulent boundary layer using grit 36 (Gul \& Ganapathisubramani 2021) and (Squire et al. 2016), and turbulent TC flow using grit 36 (present). ES is the effective slope defined as $|\overline{\mathrm{d} k / \mathrm{d}(r \theta)}|$ (Napoli, Armenio \& De Marchis 2008).

rough wall TC experiments is consistent with the empirical trend given for the sandpaper used in rough wall turbulent BL flow analysis. It was hypothesized by Chung et al. (2021) that for fixed skewness, $k_{s} / k$ versus ES increases with increasing ES, exhibits a maximum, and then will decrease with further increasing ES. The results in figure 6 support this hypothesis. Whether the deviation originates from the difference between TC and canonical systems without curvature, or originates from the different surface statistics (e.g. the ES for the present surface is higher, which indicates a denser surface), remains to be resolved.

\section{The constant angular momentum region in the bulk}

Thus far, we have discussed the velocity profiles of the inner cylinder boundary layer, i.e. $y^{+}<0.65 L_{c}^{+}$. By means of matching this profile to the bulk velocity profile at the boundary layer height, one can derive the relationship between the torque $N u_{\omega}(T a)$ and the velocity of the inner cylinder (Berghout et al. 2020; Cheng et al. 2020). For smooth wall inner cylinder rotating turbulent TC flow, it is well known that the angular momentum in the bulk $\left(M_{b}\right)$ is constant (Wendt 1933; Townsend 1976), and, in fact, very close to half the inner cylinder angular momentum $\left(M_{i}=\omega_{i} r_{i}^{2}\right), M_{b}=0.5 M_{i}$, for a stationary outer cylinder. For rough wall TC flow however, and especially for asymmetric roughness when the inner cylinder is of a different roughness height than the outer cylinder, the exact value of $M_{b}$ is a priori unknown. However, what was shown is that for very rough walls, the bulk azimuthal velocity profile is shifted towards the rough cylinder (Zhu et al. 2017, 2018; Berghout et al. 2019).

If the bulk region velocity conforms to a constant angular momentum, it should match the angular momentum at the edge of the BL $r=r_{i}+\delta_{r}$, where $\delta_{r}=0.65 L_{c}$. The momentum ratio $\left(M_{b} / M_{i}\right)$ is the angular momentum in the bulk over the angular momentum of the inner cylinder

$$
\frac{M_{b}}{M_{i}}=\frac{\left.\omega\right|_{y=\delta_{r}}\left(r_{i}+\delta_{r}\right)^{2}}{\omega_{i} r_{i}^{2}}
$$




\section{P. Berghout and others}
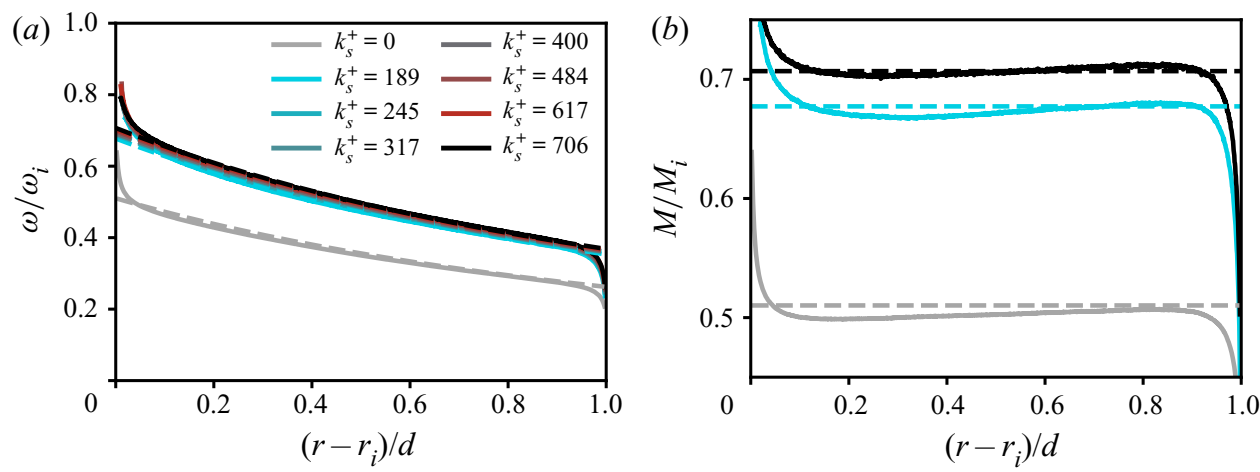

Figure 7. Bulk velocity profiles. (a) The mean angular velocity normalized with the inner velocity $\omega / \omega_{i}$ versus the radius $\left(r-r_{i}\right) / d$ normalized with the gap width $d$. The profiles for different roughness heights $k_{s}^{+}$are compared. The bulk profile is strongly shifted towards the rough inner cylinder, as the roughness there enhances the coupling between the inner BL and the bulk, similarly as the ribs have done in Zhu et al. (2018). (b) The angular momentum $M$ normalized with the inner cylinder angular momentum $M_{i}$. Solid lines are the PIV results and dashed lines $\left(M_{b} / M_{i}\right)$ are calculated from (7.1). The colours are the same in both figures. The grey line is the smooth wall profile at $T a=6.2 \times 10^{12}$, obtained from Huisman et al. (2013).

where $\left.\omega\right|_{y=\delta_{r}}=\omega_{\tau, i}\left(\omega_{i}^{+}-\omega_{r}^{+}\left(y^{+}=\delta_{r}^{+}\right)\right)$, and we use the velocity profile of the rough inner cylinder BL, figure 3 and (3.3), $\Delta \omega^{+}, \omega_{r}^{+}\left(y^{+}=\delta_{r}^{+}\right)=(1 / \lambda) \log \delta_{r}^{+}+(1 / \kappa-$ $1 / \lambda) \log L_{c, r}^{+}+1.0-\Delta \omega^{+}$. Figure 7 compares the result from (7.1) (dashed line) with the experimentally obtained velocity profiles (solid lines), which demonstrates agreement between the calculated and the measured profiles. This supports the assumption that the rough-wall velocity profiles also conform to a constant angular momentum in the bulk. Finally, we point out that the 'undershooting' and 'overshooting' of the profiles in the bulk, i.e. the slight increase in $M$ with increasing $r$, is likely an effect of the turbulent Taylor vortices, and is therefore expected to depend on the height coordinate $z$ (Huisman et al. 2014). This arises from the detaching plumes which are transported to the other side of the gap by the Taylor rolls. Similar overshooting is well known from temperature profiles in turbulent Rayleigh-Bénard flow (Tilgner, Belmonte \& Libchaber 1993; Ahlers, Grossmann \& Lohse 2009).

\section{Calculation of $N u_{\omega}(T a)$ and $C_{f}(R e)$}

Because the angular momentum in the bulk is, to a good approximation, constant, we can match the angular momentum of the inner cylinder BL at the BL height with the angular momentum of the outer cylinder BL at the BL height, i.e. $M\left(\delta_{i, r}\right)=M\left(\delta_{o, s}\right)$. This approach is based on the matching of the BL and bulk velocity profiles in the recent CPS model (Cheng et al. 2020). Subscripts $(i, o$ ) refer to inner cylinder and outer cylinder BL quantities, where subscripts $(s, r)$ refer to smooth and rough wall quantities, and $\delta=$ $0.65 L_{c}$ for the inner cylinder and outer cylinder (rough and smooth) so that $\delta_{i, r}^{+}=\alpha L_{c, i, r}^{+}$, $\delta_{o, s}^{+}=\alpha L_{c, o, s}^{+}$with $\alpha=0.65$. The matching argument becomes

$$
\left(r_{i}+\delta_{i, r}\right)^{2} \omega_{\tau, i} \omega_{I C}^{+}\left(\delta_{i, r}^{+}\right)=\left(r_{o}-\delta_{o, s}\right)^{2} \omega_{\tau, o} \omega_{O C}^{+}\left(\delta_{o, s}^{+}\right),
$$




\section{Taylor-Couette flow with rough surfaces}

where we realize that $\omega_{\tau, o}=\eta^{2} \omega_{\tau, i}$. We substitute the BL equations for respectively rough and smooth walls into (8.1) and obtain

$$
\begin{gathered}
\left(r_{i}+\delta_{i, r}\right)^{2} \omega_{\tau, i}\left(\omega_{i}^{+}-\frac{1}{\lambda} \log \left(\delta_{i, r}^{+}\right)-\left(\frac{1}{\kappa}-\frac{1}{\lambda}\right) \log \left(L_{c, i, r}^{+}\right)-C_{i}+\Delta \omega^{+}\right) \\
=\left(r_{o}-\delta_{o, s}\right)^{2} \omega_{\tau, o}\left(\frac{1}{\lambda} \log \left(\delta_{o, s}^{+}\right)+\left(\frac{1}{\kappa}-\frac{1}{\lambda}\right) \log \left(L_{c, o, s}^{+}\right)+C_{o}\right) .
\end{gathered}
$$

The rough wall, inner cylinder BL height $\delta_{i, r}^{+}$and the velocity shift $\Delta \omega^{+}$are functions of the sand grain size $k_{s}^{+}$. This makes the matching equation more involved, compared with the smooth wall case (Berghout et al. 2020; Cheng et al. 2020).

Following Cheng et al. (2020), we now rewrite the equation in terms of $R e_{\tau, i}$ and $R e_{i}$. The inner cylinder angular velocity becomes

$$
\omega_{i}^{+}=\frac{R e_{i}}{2 R e_{\tau, i}}
$$

The equivalent sand grand size is

$$
k_{s}^{+}=2 \frac{k_{s}}{d} R e_{\tau, i}=\epsilon \operatorname{Re}_{\tau, i} .
$$

The fully rough asymptote from (5.1) can now be rewritten as

$$
\Delta \omega^{+}=\frac{1}{\kappa} \log \left(\epsilon R e_{\tau, i}\right)+A-B .
$$

The inner cylinder, rough wall, BL height $\delta_{i, r}^{+}$is rewritten from $\delta_{i, r}^{+}=\alpha L_{c, i, r}^{+}$as

$$
\delta_{i, r}^{+}=\frac{2 \alpha \eta R e_{\tau, i}}{\kappa(1-\eta) \mathcal{Z}} ; \quad \text { with } \mathcal{Z}=\left(\frac{\operatorname{Re}_{i}}{2 \operatorname{Re}_{\tau, i}}+\frac{1}{\kappa} \log \left(\epsilon \operatorname{Re}_{\tau, i}\right)+A-B\right) .
$$

The outer cylinder, smooth wall, BL height $\delta_{o, s}^{+}$is rewritten from $\delta_{o, r}^{+}=\alpha L_{c, o, r}^{+}$as

$$
\delta_{o, s}^{+}=\frac{4 \alpha \eta^{2} R e_{\tau, i}^{2}}{\kappa(1-\eta) R e_{i}}
$$

We can now substitute (8.3)-(8.7) into (8.2), and obtain

$$
\begin{aligned}
(1+ & \left.\frac{\alpha}{\kappa \mathcal{Z}}\right)^{2}\left(\frac{R e_{i}}{2 R e_{\tau, i}}-\frac{1}{\lambda} \log \left(\frac{2 \alpha \eta R e_{\tau, i}}{\kappa(1-\eta) \mathcal{Z}}\right)\right. \\
& \left.-\left(\frac{1}{\kappa}-\frac{1}{\lambda}\right) \log \left(\frac{2 \eta R e_{\tau, i}}{\kappa(1-\eta) \mathcal{Z}}\right)+\frac{1}{\kappa} \log \left(\epsilon \operatorname{Re}_{\tau, i}\right)+A-B-C_{i}\right) \\
= & \left(1-\frac{2 \alpha \eta R e_{\tau, i}}{\kappa \operatorname{Re}_{i}}\right)^{2}\left(\frac{1}{\lambda} \log \left(\frac{4 \alpha \eta^{2} R e_{\tau, i}^{2}}{\kappa(1-\eta) \operatorname{Re}_{i}}\right)+\left(\frac{1}{\kappa}-\frac{1}{\lambda}\right) \log \left(\frac{4 \eta^{2} R e_{\tau, i}^{2}}{\kappa(1-\eta) \operatorname{Re}_{i}}\right)+C_{o}\right) .
\end{aligned}
$$

This implicit equation can be solved numerically to obtain $\operatorname{Re}_{\tau, i}\left(R e_{i}\right)$ with parameters $C_{i}=1.0, C_{o}=2.0, A=5.0, B=8.5, \kappa=0.39$ (in accordance with Huisman et al. 2013; Berghout et al. 2020), $\lambda=0.64, \alpha=0.65$ for these experiments, $\eta=0.714$ and 
(a)

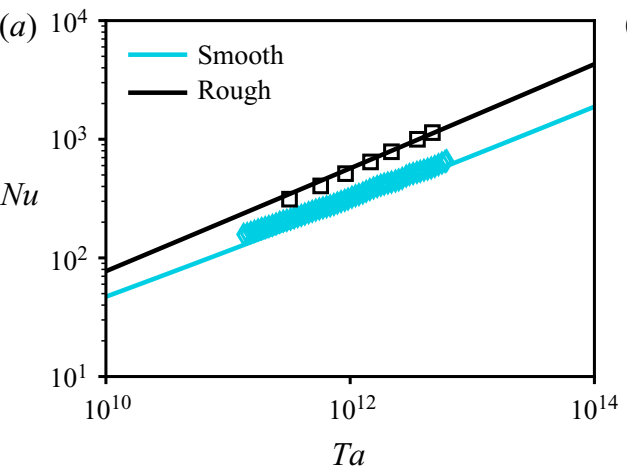

(b)

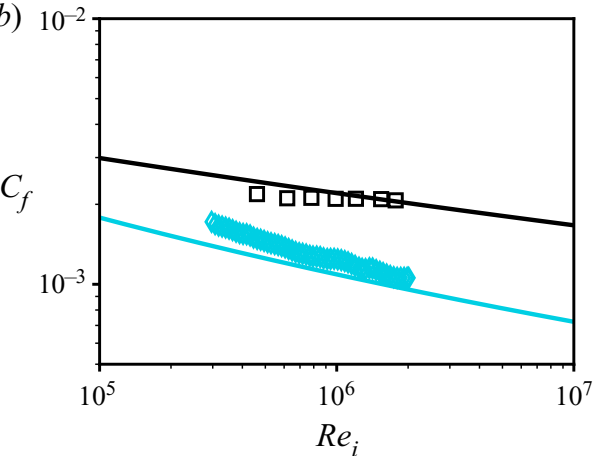

Figure 8. Global response of the rough $k_{s} / d=0.012$ and smooth wall TC turbulence. (a) Nusselt number $N u_{\omega}=\left(2 R e_{\tau}^{2} \eta(1+\eta)\right) / R e_{i}$ versus $T a$. (b) Friction factor $C_{f}=8 R e_{\tau}^{2} / R e_{i}^{2}$ versus $R e_{i}$. Blue diamonds are the smooth wall experiments of van Gils et al. (2011b), where the solid blue line shows the theory of Berghout et al. (2020) for smooth wall TC turbulence. Black squares are the rough inner cylinder measurements from the present work. The solid black line is (8.8).

$\epsilon=2(0.9694 / 78.2)=0.024$. Finally, by means of (1.3)-(2.1), we express the result $R e_{\tau, i}\left(R e_{i}\right)$ into $N u_{\omega}(T a)$ and $C_{f}\left(R e_{i}\right)$, respectively.

Figure 8 presents the final result, together with the experimental data from smooth walls (van Gils et al. 2011b) and with the equation for smooth wall TC (Berghout et al. 2020) (grey). The black open squares represent the fully rough inner cylinder rotating TC experiments presently. The black solid line is our calculation from (8.8). We emphasize that no fitting parameters were used. All parameters find their origin in the velocity profiles, and originate from the slopes of the logarithmic velocity profiles $\left(\kappa^{-1}, \lambda^{-1}\right)$, the offset of the smooth velocity profile $\left(A, C_{i}, C_{o}\right)$ or the BL thickness fit for smooth walls $\alpha$. This reflects that all parameters are universal for all radius ratios, and cannot and need not be 'tuned'. Regarding the quantification of the accuracy and repeatability of the torque measurements, we carried out 6 individual time series measurements of the torque at a fixed $R e=0.99 \times 10^{6}$. We find that the mean standard deviation of the individual time series of the torque, binned over 1 min intervals, was 1.72, which is larger than the error in the torque sensor typically expressed by manufacturers as a percentage of the maximum rated load. The maximum spread in the mean Nusselt numbers among the different measurements was 5.54. Both numbers were smaller than the marker size in figure $8(a)$.

The agreement between (8.8) and the experimental data is convincing. The mean absolute error was only $4.9 \%$, and for the three highest Taylor numbers, the error was less than $2 \%$. This implies that from straightforward measurements of the torque, for given inner cylinder rotation speed, we can calculate the value of $k_{s}$ with a reasonable accuracy. This means that the TC facility can potentially be used for direct and fast measurements of surface drag properties, as characterized by $k_{s}$.

\section{Discussion}

In the foregoing sections, we have shown that the TC set-up can be employed to study the drag of rough surfaces. In particular, we have investigated the necessary scale separation, the functional form of the velocity profiles and, from the velocity profiles, the calculation of $N u_{\omega}(T a)$. Here we address some final implications of the experiments and modelling ideas. 


\section{Taylor-Couette flow with rough surfaces}

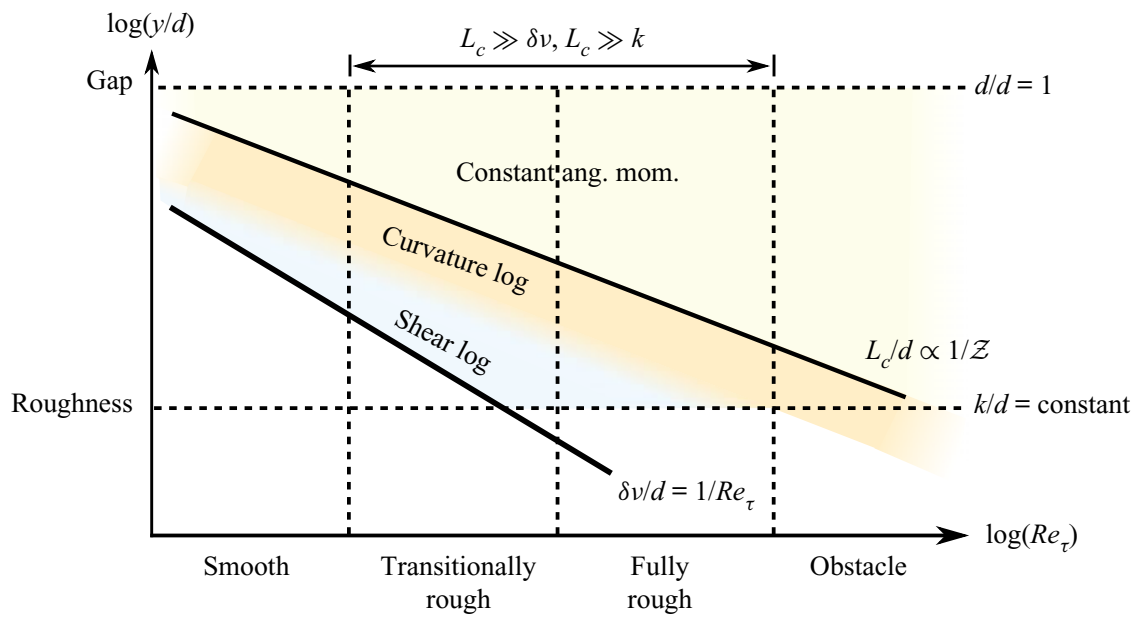

Figure 9. Schematic of the various rough TC flow regimes and their relationships with the mean velocity profile.

The calculation of $N u_{\omega}(\mathrm{Ta})$ mostly follows the idea of matching velocity profiles of Cheng et al. (2020). However, Cheng et al. (2020) assumed that the bulk angular momentum is always 0.5 times the inner cylinder angular momentum. In $\S 7$, we have shown that this is not the case when the drag of the inner cylinder is different from the drag of the outer cylinder. Consequently, we matched the angular momentum of the IC BL with the bulk at the boundary layer height, and calculated the bulk angular momentum as a function of the roughness height (see figure 7 and (7.1)).

In $\S 5$, we showed that the rough IC BL velocity profile follows, to a good approximation, the fully rough asymptote. Hence, we conclude that the turbulent flow occupies the fully rough regime. However, the friction factor $C_{f}$ does not exhibit fully rough behaviour, i.e. $C_{f}\left(R e_{i}\right)$ is not constant, see figure $8(b)$. This arises from the fact that only the IC is rough and the outer cylinder is still smooth. Much like the experiments of Zhu et al. (2018), who found that only when both cylinders are rough and pressure drag dominates viscous drag at both cylinders is $\tau_{w} \propto R e_{i}^{2}$, i.e. $C_{f}\left(R e_{i}\right)=$ Constant. If both cylinders would be rough, we could modify the right hand side of (8.2) and match two rough angular momentum profiles. This is left for future research.

Finally, we note that a limit exists for which the modelling, as discussed in this paper, would no longer work. At that limit, the dispersive fluctuations of the rough wall are not constrained to the shear logarithmic region, i.e. $h_{r} /\left(0.20 L_{c}\right)>1$ (with $h_{r}$ as the roughness sublayer height) and the flow enters the obstacle regime, see figure 9. However, we realize that this limit is far away in terms of $R e_{\tau}$, as $L_{c} / d \propto 1 / \mathcal{Z} \propto 1 /\left(R e_{i} / \operatorname{Re}_{\tau}+\log \left(R e_{\tau}\right)\right)$. For the current system configuration, we calculate that $h_{r}>0.20 L_{c}$ for $\operatorname{Re}_{\tau}>O\left(10^{7}\right)$, with $h_{r} \approx 2.5 k_{s}$, see Berghout et al. (2019). In fact, the obstacle regime might itself consist of two varying regimes. One where $0.20 L_{c}<h_{r}<L_{c}$, and the roughness penetrates the curvature logarithmic region. Then a second regime where $h_{r}>L_{c}$ and the roughness penetrates the bulk.

\section{Summary, conclusions and outlook}

We conducted experiments of inner cylinder rotating (and stationary outer cylinder) Taylor-Couette (TC) turbulence with a rough inner cylinder and a smooth outer cylinder. 


\section{P. Berghout and others}

We measured the torque and, by means of PIV, the mean angular velocity profiles. The rough surface consisted of P36 industrial grade sandpaper, where the roughness height $k=6 k_{\sigma}$, with $k_{\sigma}$ as the standard deviation of the roughness height, over the gap width $d$ was $k / d=0.014$. The roughness height $k$ was much larger than the viscous length scale $\delta_{v}$, such that $k / \delta_{v}=204-762$. The velocity shift of the rough wall angular velocity profiles, compared with the reference smooth wall, in the 'log-law region', was $\Delta \omega^{+}>9$ over the whole range of $4.6 \times 10^{5}<R e_{i}<1.77 \times 10^{6}$. Hence, the sandpaper was hydrodynamically fully rough. Furthermore, the roughness height $k<0.090 L_{c}$, where $0.20 L_{c}$ is the height (Berghout et al. 2020) that separates the region in the BL where production of turbulence is dominated by shear from the region in the BL where production of turbulence is affected by streamwise curvature.

Using the mean azimuthal velocity profiles, we found that the slope of the fully rough asymptote, characterized by $\kappa=0.34 \pm 0.02$, was similar to previous findings in flat-plate BLs $\kappa \approx 0.38$. Also, the value of the equivalent sand grain roughness height $k_{s}$ compared reasonably well with those found for sandpaper in flat plate BLs (Flack et al. 2007; Squire et al. 2016).

Finally, to obtain the relationship between the dimensionless torque and dimensionless driving of the system $N u_{\omega}(\mathrm{Ta})$, we employed a matching argument between the inner cylinder BL rough mean angular momentum profile at the inner cylinder BL height, and the smooth outer cylinder BL mean angular momentum profile at the outer cylinder BL height, based on the CPS model of Cheng et al. (2020), see also Berghout et al. (2020). To justify this, we first showed that for a rough wall inner cylinder, a region of constant angular momentum exists in the bulk. We find a convincing overlap between the calculated value of the torque (or wall shear stress), and the experimentally measured values of the torque, with a mean absolute error of $4.9 \%$.

These findings indicate that the turbulent TC facility can be a valuable set-up for characterizing the turbulent drag properties of any rough surface. Direct and straightforward measurements of the torque can now be translated to a value of the equivalent sand grain roughness height $k_{s}$. It seems that the value of $k_{s}$ found in TC is similar to the value of $k_{s}$ found in flat-plate BLs.

As an outlook to future work, we propose that more studies in both turbulent flat-plate BLs and turbulent TC flow, with identical rough surfaces, are carried out to further compare the drag properties of these surfaces. Further unanswered questions include the effects of even more considerable roughness penetrating the curvature-affected logarithmic regime of the $\mathrm{BL}$, which is related to finding the slope of the fully rough asymptote in that region. This could also be achieved by employing a TC set-up with a lower radius ratio $\eta$, thus increasing curvature effects.

Acknowledgements. We would like to thank B. Benschop, M. Bos and G.W. Bruggert for their technical support, Y.A. Lee for his support in the lab, and M.A. Bruning for discussions. We also thank D. Pullin for his generous and profound comments on the manuscript.

Funding. This study was funded by the Netherlands Organisation for Scientific Research (NWO) through the Multiscale Catalytic Energy Conversion (MCEC) research center and the GasDrive project 14504, by the European Research Council (ERC) Advanced Grant 'Droplet Diffusive Dynamics', and by the Priority Programme SPP 1881 Turbulent Superstructures of the Deutsche Forschungs-gemeinschaft.

Declaration of interests. The authors report no conflicts of interest.

\footnotetext{
Author ORCIDs.

(D) Pieter Berghout https://orcid.org/0000-0002-7231-3132;

Pim A. Bullee https://orcid.org/0000-0001-7602-7726;
} 

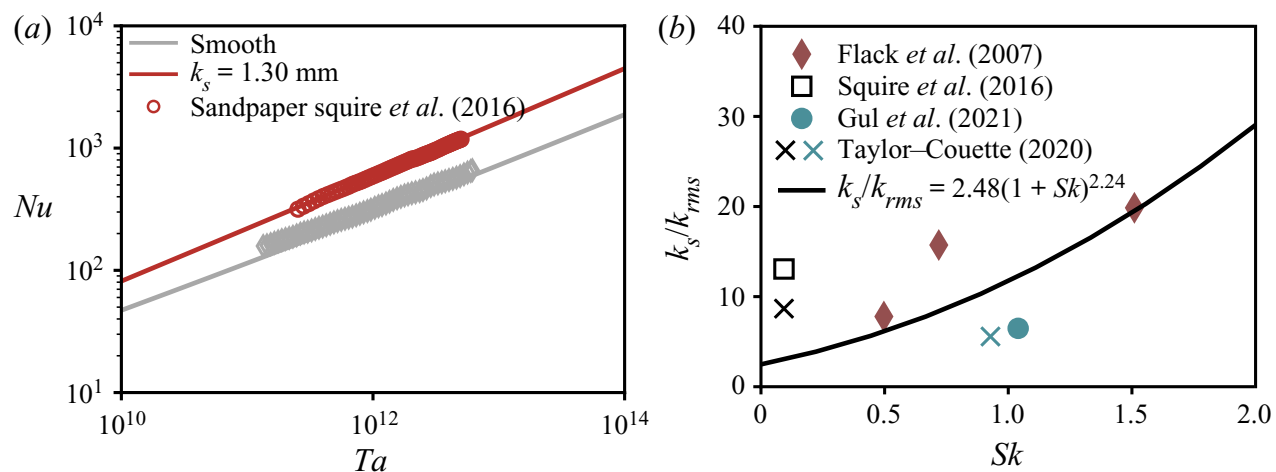

Figure 10. (a) Torque measurements of the sandpaper of Squire et al. (2016) in TC. Solid red line is the fit of (8.8) to the torque data, with $k_{s}$ the only fitting parameter. (b) Relationship between the equivalent sand grain roughness height divided by the root-mean-square height $k_{s} / k_{\sigma}$, and the skewness parameter $S k$ of different sandpaper surfaces. The solid black line is the empirical correlation for $S k>0$ from Flack et al. (2020). Data from turbulent boundary layer flow using grit $(12,24$ and 80) sandpaper (Flack et al. 2007), of which the surface statistics are listed by Flack \& Schultz (2010), turbulent boundary layer using grit 36 (Squire et al. 2016; Gul \& Ganapathisubramani 2021) and turbulent TC flow using grit 36 (present). The black cross is the Squire sandpaper, whereas the lighter colour cross represents the VSM, ceramic industrial grade, sandpaper.

Di Thomas Fuchs https://orcid.org/0000-0002-4897-8484;

(1) Sven Scharnowski https://orcid.org/0000-0002-6452-2954;

(1) Daniel Chung https://orcid.org/0000-0003-3732-364X;

(1) Detlef Lohse https://orcid.org/0000-0003-4138-2255;

Sander G. Huisman https://orcid.org/0000-0003-3790-9886.

Author contributions. P.B. and P.A.B. are joint first authors.

\section{Appendix}

In the manuscript, we have described the experimental difficulties that we encountered with the sandpaper surface of Squire et al. (2016) ('Squire sandpaper'), i.e. the sandpaper detached from the inner cylinder at high $R e_{i}$. Nonetheless, we studied the torque data of the one successful experiment that was done before the sandpaper surface ruptured from the inner cylinder, and did not include a PIV measurement. From this torque data, which comes from just one experiment, we can, by means of (8.8), calculate the value of $k_{s}$. We are cautious in doing so. First, the torque is obtained from one experiment only. Second, for the Squire sandpaper, we did not measure the velocity profiles that would directly give us $k_{s}$ and would inevitably be more precise. Nonetheless, we show in figure $10(a)$ the fit of the (8.8) from the manuscript with the torque data of the Squire sandpaper surface. The fit gives $k_{s}=1.30 \mathrm{~mm}$. In figure $10(b)$, we plot $k_{s} / k_{r m s}$ versus the skewness for all sandpaper surfaces. We find that $k_{s} / k_{r m s}$ is indeed higher for the Squire sandpaper surface as measured in TC (where we take the skewness value from Squire et al. 2016), as compared to the sandpaper surface that we employ in the rest of our experiments (VSM, ceramic industrial grade), despite the lower skewness for the Squire sandpaper. Also, we find that the value of $k_{s}$ is rather close to that of Squire et al. (2016).

In the end, the conclusion of the paper remains the same. We have shown the existence of a fully rough asymptote in TC turbulence and how to calculate the value of $k_{s}$ from the torque data. The value of $k_{s}$ as measured in TC appears to be similar to that of 


\section{P. Berghout and others}

$k_{s}$ measured in BL turbulence for identical surfaces. However, only more careful and structured experimental work with all kinds of surfaces can answer whether the values of $k_{s}$ for the different systems will ultimately be the same for identical surfaces, or whether differences remain.

\section{REFERENCES}

Ahlers, G., Grossmann, S. \& Lohse, D. 2009 Heat transfer and large scale dynamics in turbulent Rayleigh-Bénard convection. Rev. Mod. Phys. 81, 503.

Baars, W.J., Squire, D.T., Talluru, K.M., Abbassi, M.R., Hutchins, N. \& Marusic, I. 2016 Wall-drag measurements of smooth- and rough-wall turbulent boundary layers using a floating element. Exp. Fluids 57, 57-90.

Bakhuis, D., Ezeta, R., Berghout, P., Bullee, P.A., Tai, N.C., Chung, D., Verzicco, R., Lohse, D., Huisman, S.G. \& SUN, C. 2020 Controlling secondary flow in Taylor-Couette turbulence through spanwise-varying roughness. J. Fluid Mech. 883, A15.

VAn Den Berg, T.H., Doering, C.R., Lohse, D. \& Lathrop, D. 2003 Smooth and rough boundaries in turbulent Taylor-Couette flow. Phys. Rev. E 68, 036307.

Berghout, P., Verzicco, R., Stevens, R.J.A.M., Lohse, D. \& Chung, D. 2020 Calculation of the mean velocity profile for strongly turbulent Taylor-Couette flow and arbitrary radius ratios. J. Fluid Mech. 905, A11.

Berghout, P., Zhu, X., Chung, D., Verzicco, R., Stevens, R.J.A.M. \& Lohse, D. 2019 Direct numerical simulations of Taylor-Couette turbulence: the effects of sand grain roughness. J. Fluid Mech. 873, 260-286.

BRADSHAW, P. 1969 The analogy between streamline curvature and buoyancy in turbulent shear flow. J. Fluid Mech. 36, 177-191.

Cadot, O., Couder, Y., Daerr, A., Douady, S. \& Tsinober, A. 1997 Energy injection in closed turbulent flows: stirring through boundary layers versus inertial stirring. Phys. Rev. E 56, 427-433.

Cheng, W., Pullin, D.I. \& Samtaney, R. 2020 Large-eddy simulation and modeling of Taylor-Couette flow with an outer stationary cylinder. J. Fluid Mech. 890, A17.

Chung, D., Hutchins, N., Schultz, M.P. \& Flack, K.A. 2021 Predicting the drag of rough surfaces. Апnи. Rev. Fluid Mech. 53, 439-471.

Clauser, F.H. 1954 Turbulent boundary layers in adverse pressure gradients. J. Aeronaut. Sci. 21, 91-108.

Eckhardt, B., Grossmann, S. \& LohSE, D. 2007 Torque scaling in turbulent Taylor-Couette flow between independently rotating cylinders. J. Fluid Mech. 581, 221-250.

FLACK, K.A. \& SCHULTZ, M.P. 2010 Review of hydraulic roughness scales in the fully rough regime. Trans. ASME J. Fluids Engng 132, 041203.

FLACK, K.A., SChUlTZ, M.P. \& BARROS, J.M. 2020 Skin friction measurements of systematically-varied roughness: probing the role of roughness amplitude and skewness. Flow Turbul. Combust. 104, 317-329.

FlaCK, K.A., SChULTZ, M.P. \& CONNELLY, J.S. 2007 Examination of a critical roughness height for outer layer similarity. Phys. Fluids 19 (9), 095104.

Forooghi, P., Stroh, A., Magagnato, F., Jakirlić, S. \& Frohnapfel, B. 2017 Toward a universal roughness correlation. Trans. ASME J. Fluids Engng 139, 121201.

van Gils, D.P.M., Bruggert, G.W., Lathrop, D.P., Sun, C. \& Lohse, D. 2011a The Twente turbulent Taylor-Couette $\left(T^{3} C\right)$ facility: strongly turbulent (multi-phase) flow between independently rotating cylinders. Rev. Sci. Instrum. 82, 025105.

van Gils, D.P.M., Huisman, S.G., Bruggert, G.W., Sun, C. \& Lohse, D. $2011 b$ Torque scaling in turbulent Taylor-Couette flow with co- and counter-rotating cylinders. Phys. Rev. Lett. 106, 024502.

van Gils, D.P.M., Huisman, S.G., Grossmann, S., Sun, C. \& Lohse, D. 2012 Optimal Taylor-Couette turbulence. J. Fluid Mech. 706, 118-149.

Grossmann, S., LohSE, D. \& Sun, C. 2014 Velocity profiles in strongly turbulent Taylor-Couette flow. Phys. Fluids 26, 025114.

Grossmann, S., Lohse, D. \& Sun, C. 2016 High Reynolds number Taylor-Couette turbulence. Annu. Rev. Fluid Mech. 48, 53-80.

Gul, M. \& Ganapathisubramani, B. 2021 Revisiting rough-wall turbulent boundary layers over sand-grain roughness. J. Fluid Mech. 911, A26.

HAMA, F.R. 1954 Boundary-layer characteristics for smooth and rough surfaces. Trans. Soc. Nav. Archit. Mar. Engrs 62, 333-358. 


\section{Taylor-Couette flow with rough surfaces}

Huisman, S.G., Scharnowski, S., Cierpka, C., Kähler, C.J., Lohse, D. \& Sun, C. 2013 Logarithmic boundary layers in strong Taylor-Couette turbulence. Phys. Rev. Lett. 110, 264501.

Huisman, S.G., VAn der Veen, R.C.A., Sun, C. \& Lohse, D. 2014 Multiple states in highly turbulent Taylor-Couette flow. Nat. Commun. 5, 3820.

JiméneZ, J. 2004 Turbulent flows over rough walls. Annu. Rev. Fluid Mech. 36, 173-196.

KÄhler, C.J., Scharnowski, S. \& CierpKA, C. 2012 On the resolution limit of digital particle image velocimetry. Exp. Fluids 52, 1629-1639.

Kähler, C.J., Scholz, U. \& ORTMAnns, J. 2006 Wall-shear-stress and near-wall turbulence measurements up to single pixel resolution by means of long-distance micro-PIV. Exp. Fluids 41, 327-341.

Monin, A.S. \& Yaglom, A.M. 1975 Statistical Fluid Mechanics. MIT Press.

NApoli, E., Armenio, V. \& DE MARChis, M. 2008 The effect of the slope of irregularly distributed roughness elements on turbulent wall-bounded flows. J. Fluid Mech. 613, 385-394.

NikuRAdse, J. 1933 Strömungsgesetze in rauhen Rohren (trans. flow laws in rough pipes). Forsch. Arb. Ing. Wes. 361.

Овикноv, A.M. 1971 Turbulence in an atmosphere with a non-uniform temperature. Boundary-Layer Meteorol. 2, 7-29.

Ostilla-Mónico, R., Verzicco, R., Grossmann, S. \& Lohse, D. 2015 The near-wall region of highly turbulent Taylor-Couette flow. J. Fluid Mech. 788, 95-117.

Pope, S.B. 2000 Turbulent Flow. Cambridge University Press.

Raupach, M.R., Antonia, R.A. \& Rajagopalan, S.S. 1991 Rough-wall turbulent boundary layers. Trans. ASME J. Fluids Engng 44 (1), 1-25.

SCHARNOWSKI, S., HAIN, R. \& KäHLER, C.J. 2012 Reynolds stress estimation up to single-pixel resolution using PIV-measurements. Exp. Fluids 52, 985-1002.

Squire, D.T., Morrill-Winter, C., Hutchins, N., Schultz, M.P., Klewicki, J.C. \& Marusic, I. 2016 Comparison of turbulent boundary layers over smooth and rough surfaces up to high Reynolds numbers. J. Fluid Mech. 795, 210-240.

TAYLOR, G.I. 1923 Experiments on the motion of solid bodies in rotating fluids. Proc. R. Soc. Lond. A 104, 213-218.

Tilgner, A., Belmonte, A. \& Libchaber, A. 1993 Temperature and velocity profiles of turbulence convection in water. Phys. Rev. E 47, R2253-R2256.

Townsend, A.A. 1976 The Structure of Turbulent Shear Flow, 2nd edn. Cambridge University Press.

van der Veen, R.C.A., Huisman, S.G., Merbold, S., Harlander, W., Egbers, C., Lohse, D. $\&$ Sun, C. 2016 Taylor-Couette turbulence at radius ratio $\eta=0.5$ : scaling, flow structures and plumes. J. Fluid Mech. 799, 334-351.

Verschoof, R.A., Zhu, X., Bakhuis, D., Huisman, S.G., Verzicco, R., Sun, C. \& Lohse, D. 2018 Rough-wall turbulent Taylor-Couette flow: The effect of the rib height. Eur. Phys. J. E 41 (10), 125.

WENDT, F. 1933 Turbulente Strömungen zwischen zwei rotierenden Zylindern. Ingenieurs-Archiv. 4, 577-595.

Zhu, X., Verschoof, R.A., Bakhuis, D., Huisman, S.G., Verzicco, R., Sun, C. \& Lohse, D. 2018 Wall roughness induces asymptotic ultimate turbulence. Nat. Phys. 14, 417-423.

ZHU, X., VERZICCO, R. \& LOHSE, D. 2017 Disentangling the origins of torque enhancement through wall roughness in Taylor-Couette turbulence. J. Fluid Mech. 812, 279-293. 